\title{
Social responsiveness and physical space as determinants of agonistic behavior in Betta splendens
}

\author{
NANCY WOODARD CAIN, CYNTHIA JESSEN, and MICHAEL FLANAGAN \\ Bucknell University, Lewisburg, Pennsylvania 17837
}

\begin{abstract}
Two experiments examined the conditions under which Betta agonistic responding occurs by manipulating physical space and the species and social responsiveness of an opponent. In Experiment 1, both the species (conspecific or nonconspecific) and the aggressiveness (aggressive or nonaggressive) of the opponent were manipulated. The results indicated that agonistic behavior was greater with both conspecifics and aggressive opponents. Experiment 2 examined the effects of physical space and social responsiveness of the opponent on intraspecific aggressive behavior. An inverse relationship was found between physical space and the number of attacks directed toward the opponent. The results of both experiments also indicated that Betta tend to attack aggressive opponents more than nonaggressive ones. It is suggested that Betta aggress either when the individual animal is threatened or when physical space is limited.
\end{abstract}

The reinforcing aspects of aggressive behavior have been demonstrated in a number of contexts; however, there appears to be some ambiguity regarding the conditions under which aggression is thought to be reinforcing. For example, Betta often are presented with views of a displaying opponent, yet there are no direct consequences of displaying back. If the visual opponent is not socially responsive (nondisplaying), the opportunity to display ceases to be reinforcing (Baenninger, 1970; Bols, 1977). In both experimental situations, actual compat is prevented and measures of display behaviors are viewed as indications of aggressive motivation. Mice, in contrast, usually are allowed to interact freely with their opponents, yet they often are paired with a nonaggressive opponent (e.g., Tellegen \& Horn, 1972). Indeed, it has been suggested that as long as precautions are taken to "insure the safe, painless victory of the aggressor," it is easy to demonstrate a preference for attacking (Potegal, Note 1).

Adding to these ambiguities is the fact that aggression may be at most a weak reinforcer in certain contexts. For example, given the choice, rats appear to prefer social affiliation to aggression (Taylor, 1976). Furthermore, the opportunity to attack may be a much weaker reinforcer than food if the motivation for aggression is not intense (Hogan, 1974; Tellegen \& Horn, 1972).

Very little attention has been given to those factors that determine whether an animal will aggress or not,

The authors thank D. Candland, O. Floody, and A. Leshner for their critical comments on an earlier version of this manuscript, and R. Anderson. C. Barton, E. Guidi, M. Happer, and C. Lee for their technical assistance. and clearly, such information is important to the determination of the conditions under which aggression is most likely to be reinforcing. In particular, the species and the social responsiveness of the opponent, as well as the physical space available during an encounter (proximity of the opponent), are obvious factors that should determine the likelihood of aggressive behavior.

The following experiments examine some of the conditions under which Betta agonistic responding occurs by manipulating these three factors. In Experiment 1 , both the species (conspecific and nonconspecific) and the aggressiveness (aggressive or nonaggressive) of the opponent were manipulated. Experiment 2 varied factorially the effects of physical space and opponent aggressiveness on Betta agonistic behavior. In the first experiment, it was hypothesized that (1) aggressive opponents would be both attacked and displayed to more often than would nonaggressive opponents, regardless of their species, and (2) aggressive Betta would receive more attacks and displays than any other opponent.

\section{EXPERIMENT 1}

\section{Method}

Subjects. The subjects were 40 adult male Siamese fighting fish (Betta splendens) obtained from a local supplier and chosen on the basis of their ability to display to their mirror images. They were housed individually in .5-liter glass jars and were visually isolated from each other for the duration of the experiment. Water temperature was maintained between $23^{\circ}$ and $25^{\circ} \mathrm{C}$.

Apparatus. During testing, fish were netted and placed into 28.5 -liter glass aquaria, measuring $41.4 \times 26 \times 21.5 \mathrm{~cm}$, each of which housed a stimulus fish.

Procedure. The subjects were assigned randomly to one of four groups, 15 fish per group, according to the aggressiveness 
(aggressive or nonaggressive) and species (conspecific or nonconspecific) of their respective opponents. The four groups were: aggressive conspecific (AC), aggressive nonconspecific (AN), nonaggressive conspecific (NC), and nonaggressive nonconspecific (NN). Four stimulus fish were chosen, one for each of the conditions. The conspecific stimuli were two adult male Betta, one of which was aggressive (always displayed to its opponent and fought back when attacked) and one of which was nonaggressive (never displayed or attacked and always maintained a pale coloration). The nonaggressive nonconspecific was a goldfish. It did not exhibit any apparent aggressive behavior and was approximately the same size as the experimental subjects. An adult male paradise fish ( $M$. opercularis) served as the aggressive nonconspecific. It was chosen because it was observed to attack both paradise fish and Betta prior to testing and because it belongs to the same family as Betta (anabantidae).

Fish in each group were paired with the appropriate stimulus opponent during a single 10-min encounter. During these encounters, each fish was netted from its home tank and placed individually into a testing chamber housing its stimulus opponent. At the end of the encounter, the subject was returned to its home tank. The experimenter recorded the latency of the subject to first display (gill-cover extension plus fin spread), the number and duration of its displays, and the number of attacks directed toward the opponent. An attack was defined as a lunge and open-mouthed contact by the subject.

\section{Results}

The data were subjected to ANOVA with aggressiveness (aggressive or nonaggressive) and species (conspecific or nonconspecific) as independent factors. Analyses of the display data indicated that the number of displays (Figure 1) and the duration of displays (Figure 2) differed significantly between groups as a function of species $[\mathrm{F}(1,36)=6.93, \mathrm{p}<.05$; $\mathrm{F}(1,36)=13.04, \mathrm{p}<.01]$, but not as a function of aggressiveness $[F(1,36)<1, p>.05 ; F(1,36)=1.74$, $\mathrm{p}>$.05]. However, a significant interaction of species

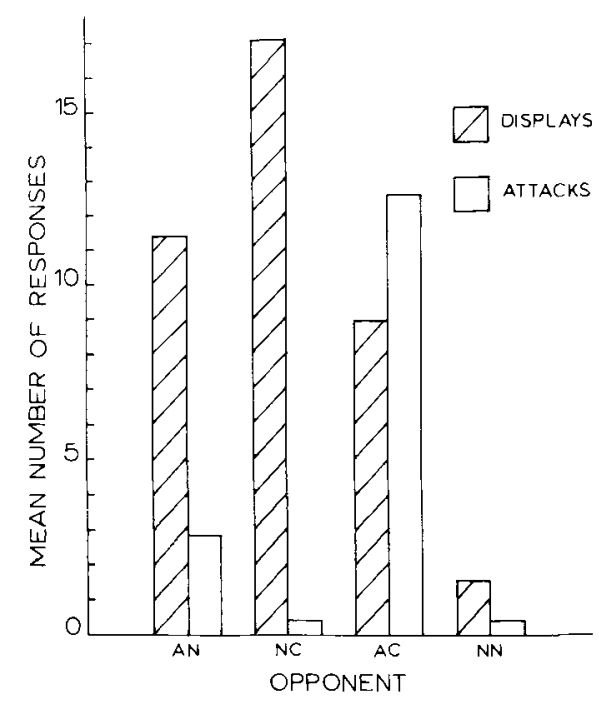

Figure 1. Mean number of displays and attacks directed loward aggressive conspecifics ( $\mathrm{AC}$ ), nonaggressive conspecifics (NC), aggressive nonconspecifics ( $A N$ ), and nonaggressive nonconspecifics (NN).

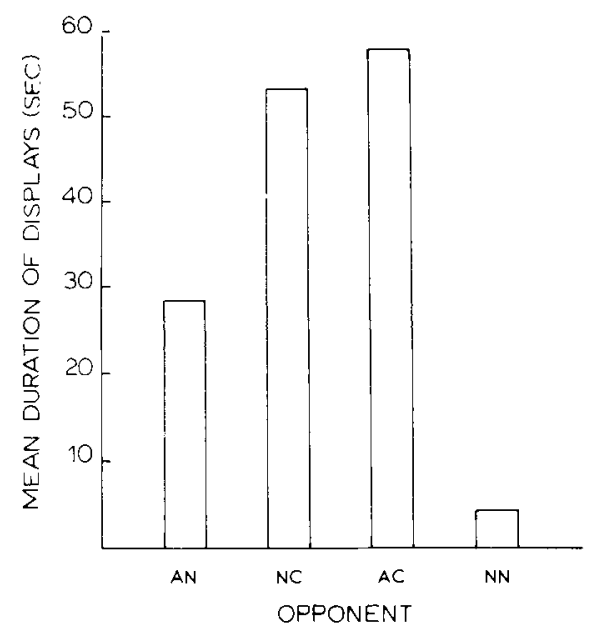

Figure 2. Mean display durations (in seconds) of experimental fish toward aggressive conspecifics $(\mathrm{AC})$, nonaggressive conspecifics (NC), aggressive nonconspecifics ( $A N)$, and nonaggressive nonconspecifics (NN).

with aggressiveness $[F(1,36)=13.3, p<.01]$ indicated that the incidence of displaying reflects the combination of both factors. Further examination of this interaction using Scheffé tests showed that fish paired with a goldfish (NN) displayed significantly less often than fish in any of the other three groups (ps < .05). Differences in display and attack behaviors for each group are summarized in Table 1. A significant interaction of aggressiveness with species $[F(1,36)=12.3, p<.01]$ also was found for the latency-to-display measure. Further analyses of this interaction (see Table 1) indicated that fish displayed more readily to conspecifics ( $\mathrm{AC}, \mathrm{NC}$ ) and paradise fish (AN) than they did to goldfish $(\mathrm{NN}, \mathrm{ps}<.05$, Scheffé).

ANOVA performed on the attack data indicated that there were significant main effects of both aggressiveness $[F(1,36)=11.34, p<.01]$ and species $[F(1,36)$ $=5.07, \mathrm{p}<.05]$, as well as a significant interaction of aggressiveness with species $[\mathrm{F}(1,36)=5.07, \mathrm{p}<.05]$. These data are presented in Figure 1. Further examination of these data indicated that Betta paired with aggressive conspecifics attacked significantly more than fish in any of the other groups (see Table 1). Also, the data indicated that fish tend to attack aggressive opponents more than nonaggressive ones and rarely attack either nonaggressive Betta (NC) or goldfish (NN).

\section{Discussion}

These results demonstrate that it is necessary to take into consideration the social responsiveness of the opponent as well as its species when studying inter- and intraspecific aggressive behavior. Indeed, three of the four measures of aggressive behavior recorded in this study depended upon the interaction 
Table 1

Summary of Significant Differences in Display and Attack Behaviors in Experiment 1

\begin{tabular}{lllll}
\hline Behavioral Measure & \multicolumn{4}{c}{ Group } \\
\hline Latency to Display & $\mathrm{AN}$ & $\mathrm{NC}$ & $\mathrm{AC}$ & $\mathrm{NN}$ \\
Number of Displays & $\mathrm{NN}$ & $\mathrm{AC}$ & $\mathrm{AN}$ & $\mathrm{NC}$ \\
\cline { 2 - 5 } Number of Attacks & $\mathrm{NN}$ & $\mathrm{NC}$ & $\overline{\mathrm{AN}}$ & $\mathrm{AC}$ \\
\hline
\end{tabular}

Note-Groups were ranked from lowest (extreme left) to highest (extreme right) mean scores and were evaluated using Scheffé tests. Any two means not underscored by the same line are significantly different. $A N=$ aggressive nonconspecific; $N C=$ nonaggressive conspecific; $A C=$ aggressive conspecific; $N N=$ nonagressive nonconspecific.

of social responsiveness and the species of the opponent. These results do not question the generality of Lorenz' (1966) claim that intraspecific aggressive behavior is more common in fish than interspecific aggression, but they suggest that it should be modified to include the social responsiveness of the opponent. Betta appear to be as aggressive as or more aggressive toward an aggressive member of another species than they are toward a nonaggressive member of their own species.

Conflicting results have been obtained in previous studies that have compared Betta inter- and intraspecific displays. The results of the present study support previous findings that Betta display as readily (Miley \& Burack, 1977) and for similar durations (Johnson \& Johnson, 1973; Miley \& Burack, 1977) to both paradise fish and other conspecifics, but they fail to support previous findings that the number of displays (Bols, 1977; Miley \& Burack, 1977) differs significantly. More important, the present studies provide data on attacks as well. These data indicate that Betta attack aggressive conspecifics, but not nonaggressive conspecifics, more often than paradise fish.

When considered alone, the intraspecific aggressive behavior data also replicate previous findings. For example, Cain, Anderson, Stein, and Jessen (1980) found that male Betta attack aggressive conspecifics significantly more often than nonaggressive conspecifics but display to both for similar amounts of time. Taken together, these results provide additional support for previous ideas that visual signals and the social responsiveness of the opponent act to facilitate or inhibit Betta attacks (Dore, Lefebvre, \& Ducharme, 1978; Johnson \& Johnson, 1973).

\section{EXPERIMENT 2}

The results of Experiment 1 confirm the findings of Johnson and Johnson (1973) and extend those results by demonstrating that both the number of displays to and the number of attacks on opponents appear to be functions of both the social responsive- ness and the species of an opponent. These findings imply that Betta attack both conspecifics and nonconspecifics when it is necessary to do so, that is, when the opponent is aggressive. Experiment 2 was designed to investigate whether a third possible factor, the amount of physical space available and the resulting proximity of the opponent, might also determine the likelihood of aggressive behavior. Relative proximity of the opponent was manipulated by varying the size of the testing tank. It was hypothesized that an inverse relationship between the incidence of agonistic behaviors and tank size would be found. In addition, it was expected, as was the case in Experiment 1, that aggressive Betta would be displayed to and attacked more than nonaggressive Betta.

\section{Method}

Subjects. The subjects were 60 adult male Siamese fighting fish (Betta splendens), obtained from a local supplier and chosen on the basis of their ability to display to their mirror images. They were housed individually in .5-liter glass jars and visually isolated from each other for the duration of the experiment. Water temperature was maintained between $23^{\circ}$ and $25^{\circ} \mathrm{C}$.

Apparatus. Combat testing was conducted in three differentsized glass aquaria: 75.7 liters (large), measuring $32 \times 41.5 \times 62 \mathrm{~cm}$; 28.5 liters (medium), measuring $41.5 \times 26 \times 21.5 \mathrm{~cm}$; and 3.8 liters (small), measuring $15.5 \times 12.5 \times 31 \mathrm{~cm}$.

Procedure. The subjects were assigned randomly to one of six groups, 10 fish per group, according to the type of stimulus (an aggressive or nonaggressive Betta) they were to be paired with and the size of the testing tank (small, medium, or large). Seven fish were chosen as stimulus opponents. One, which never displayed and always maintained a pale coloration, was designated as the nonaggressive opponent. The other six fish were designated as aggressive opponents and had to meet the criteria of always performing a display and pursuing their opponent when confronted with another male conspecific. A particular aggressive opponent was used until it failed to meet the established criteria. It then was replaced by a new aggressive opponent. Fish in each group were paired with the appropriate stimulus opponent during a single 15-min paired encounter. The testing procedure and dependent measures were the same as those described in Experiment 1.

\section{Results}

The results were subjected to ANOVA with aggressiveness (aggressive or nonaggressive) and tank size (large, medium, or small) as independent factors. Analyses of these data indicated that neither the number of displays (see Figure 3) nor the duration of displays differed as a function of tank size or the aggressiveness of the opponent (all ps $>.05$ ). In contrast, the number of attacks directed toward an opponent differed as a function of both aggressiveness $[\mathrm{F}(1,54)=38.98, \mathrm{p}<.001]$ and tank size $[\mathrm{F}(2,54)=$ $3.53, p<.05]$. As can be seen in Figure 3, the number of attacks was inversely related to tank size for both aggressive and nonaggressive opponents. A marginally significant interaction of aggressiveness with tank size $[\mathrm{F}(2,54)=2.91, \mathrm{p}=.06]$ also was found for the attack measure. Further examination of this interaction showed that fish in the three groups 


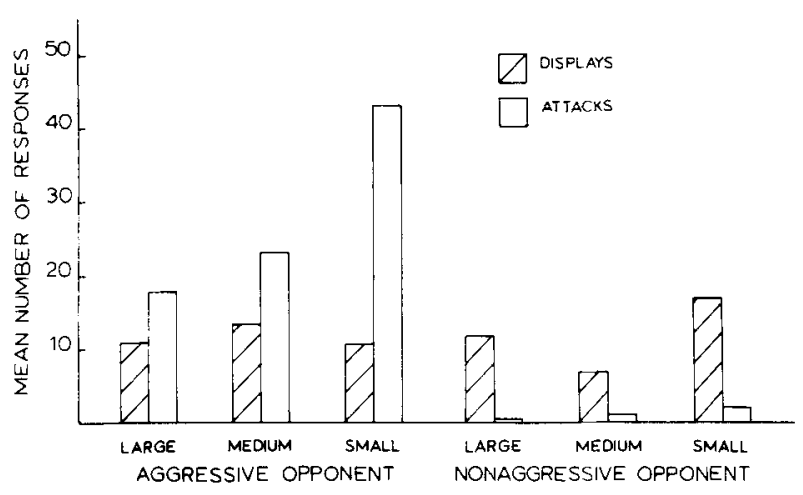

Figure 3. Mean number of displays and attacks directed toward aggressive and nonaggressive Betta in large (75.7-liter), medium (28.5-liter), and small (3.8-liter) tanks.

paired with aggressive opponents attacked significantly more often than fish in the three groups paired with nonaggressive opponents ( $\mathrm{ps}<.05$, Scheffé). In addition, no significant differences between tank size (physical space) were found for groups of fish paired with nonaggressive opponents ( $\mathrm{ps}>.05$, Scheffé); however, all differences in tank size between groups of fish paired with aggressive opponents were significant (ps $<.05$, Scheffé).

The latency to first display did not differ significantly as a function of tank size $[\mathrm{F}(2,54)<1, \mathrm{p}>.05]$, but it did differ as a function of aggressiveness $[F(1,54)=9.72, p<.01]$. Short latencies to display were evident in encounters with aggressive opponents, whereas encounters with nonaggressive opponents were characterized by much longer latencies.

\section{Discussion}

The results of Experiment 2 demonstrated that there is an inverse relationship between tank size and the number of attacks directed toward the opponent: the larger the tank size, the fewer the attacks. This same relationship, however, did not appear to hold for displays. These findings are consistent with those of other investigators (Goldstein, 1975; Johnson \& Peeke, 1972). Goldstein (1975), for example, has reported that fish continue to display in both large (426-liter) and small (6.5-liter) tank conditions, even though fighting has ceased. The similar display rates and display durations across all tank sizes found in the present study suggest that the inverse relationship between tank size and attacks is not a function of a decreased ability to detect the opponent in a larger tank. It is likely that displaying, which appears to be less aversive and energetically more efficient than attacking, continues even in large tank conditions as a means of signaling a readiness to fight, should it become necessary. Attacks, on the other hand, become less necessary as tank size increases. One explanation for this is that fish may be able to detect an increased probability of escape (Goldstein, 1975).
Another explanation might be that different degrees of proximity, together with the species and responsiveness of an opponent, could act as a sign stimulus for attacks.

Results of this experiment also showed that Betta attack aggressive opponents more often than nonaggressive ones, a relationship that was evident in Experiment 1 and in previous studies (Cain et al., 1980). Considering only the aggressive opponents, a significant decrease in attacks was found between each tank size as the tank sizes were increased. In contrast, no significant differences in attacks were found between any of the tank sizes for nonaggressive opponents, although the tendency toward an inverse relationship was evident (see Figure 3). One explanation for these findings might be that nonaggressive opponents are attacked so infrequently that it is difficult to detect any difference as a function of tank size. An alternative explanation may be that the low attack rate reflects the fact that it is not necessary or that there is no reason to attack a nonaggressive fish, regardless of the space available.

\section{GENERAL DISCUSSION}

These experiments showed that both the social responsiveness of the opponent and the physical space available during an encounter affect the aggressive behavior of Betta; fish attack aggressive opponents more often than nonaggressive ones, and the incidence of attacks appears to be an inverse function of tank size. The social responsiveness of the opponent, in particular, affects interspecific as well as intraspecific aggressive behavior, and the species of the opponent interacts with its aggressiveness.

These experiments have several important implications for studies of aggression-contingent operant performance. The typical operant paradigm involves training fish to perform some response by reinforcing it with visual presentations of display-eliciting stimuli. In this situation, actual combat is prevented, and measures of the display response are inferred to be an indication of aggressive arousal. The present studies indicate that display measures alone cannot be used synonymously with measures of aggressive behavior. For example, increasing the tank size decreased the number of attacks but did not alter significantly the number of displays. Similar findings have been reported elsewhere (Cain et al., 1980), from studies in which dominant fish showed a decrease in attacks but not displays once their opponents had submitted. Taken together, these results suggest that studies of agonistic behavior should include independent measures of both threats and attacks.

Furthermore, the ways in which aggressioncontingent operant performance has been demonstrated have not taken into consideration the probable 
factors that elicit agonistic behavior. Consider, for example, operant studies that use mirror presentation as a reinforcer (e.g., Baenninger, 1970; Baenninger \& Mattleman, 1973). In these studies, proximity (the mirror reflection of the "opponent") is held constant regardless of whether the subject displays or not. Also in these studies, the prior social experience of the subject and the social responsiveness of the opponent are confounded because the presence of a nonaggressive stimulus can only result from the subject's own reduced proclivity to display. Given that such factors as prior social experience, opponent aggressiveness, and physical space clearly affect agonistic behavior, these results are particularly important to a thorough behavioral analysis of the conditions under which aggression may be reinforcing.

\section{REFERENCE NOTE}

1. Potegal, M. Aggression and reinforcement. Unpublished manuscript, 1977.

\section{REFERENCES}

Baenninger, R. Visual reinforcement, habituation, and prior social experience of Siamese fighting fish. Journal of Comparative and Physiological Psychology, 1970, 71, 1-5.

Bafnninger, R., \& Matti.tman, R. A. Visual reinforcement: Operant acquisition in the presence of a free mirror. Animal Learning \& Behavior, 1973, 1, 303-306.

Bols, R. J. Display reinforcement in the Siamese fighting fish, Betta splendens: Aggressive motivation or curiosity? Journal of
Comparative and Phusiological Psychology, 1977, 91, 233-244.

Cain, N. W., Anderson, R., Stein, L., \& Jessen, C. Effects of prior social experience on agonistic responding by adult male Siamese fighting fish (Betta splendens). Animal Learning \& Behavior, 1980, 8, 491-496.

Dore, F., Lefebvae, L., \& Ducharme, R. Threat display in Betta splendens: Effects of water condition and type of agonistic stimulation. Animal Behaviour, 1978, 26, 738-745.

Goldstein, S. R. Observations on the establishment of a stable community of adult male and female Siamese fighting fish (Betta splendens). Animal Behaviour, 1975, 23, 179-185.

Hogan, J. A. On the choice between eating and aggressive display in the Siamese fighting fish (Betta splendens). Learning and Motivation, 1974, 5, 273-287.

JoHnson, R. N., \& Johnson, L. D. Intra- and interspecific social and aggressive behavior in the Siamese fighting fish, Betta splendens. Animal Behaviour, 1973, 21, 665-672.

Johnson, H. G., \& PrkkF. H. V. S. Patterns of intra- and interspecific aggression in labyrinth fish (Belontiidae). Behavioral Biology, 1972, 7, 335-347.

Lortan7, K. On aggression. New York: Harcourt, Brace, \& World, 1966.

Milfy, W. M., \& Burack, G. Strength of aggressive display in Siamese fighting fish (Betta splendens) toward a conspecific, an alien species (Macropodus opercularis), and a mirror image as affected by prior conspecific visual experience. Behavioral Biology, 1977. 21, 267-272.

TAYLOR, G. T. Affiliation and aggression in rats. Animal Learning \& Behavior, 1976, 4. 139-144.

Tellfegr.n, A., \& Horn, J. M. Primary aggressive motivation in three inbred strains of mice. Journal of Comparative and Phvsiological Psvchologv, 1972, 78, 297-304.

(Received for publication October 18, 1979; revision accepted March 23. 1980.) 